\title{
Numerical Forecasting over the Far East by the Double Fourier Series Method
}

\author{
By K. Gambo, K. Katow, S. Manabe and M. Aihara \\ Geophysical Institute, Tokyo University \\ (Manuscript received 25 November, 1954)
}

\begin{abstract}
The results of the numerical forecasting over the Far East is presented. In this paper, we use double Fourier series in order to calculate the Jacobian of vorticity $\zeta$ and the height of isobaric surface $Z$ and also to solve the Poisson equation. As the preliminary report we explain mainly the detailed technique of our method how to treat and apply. It is also shown that the results of numerical forecasting will be improved, if we take shorter time interval of each step. In connection with this problem, the outline of the relay-computer (FACOM-100) which is available is written briefly.
\end{abstract}

\section{§ 1. Introduction}

The purpose of this article is to present one method to calculate the Jacobian of vorticity $\zeta$ and the height of isobaric surface $Z$ and also to integrate it with respect to time by using harmonic functions.

Charney et al. [2] [3] used the extrapolated Liebman procedure to integrate the vorticity equation. On the other hand, Fjortoft [4] proposed a graphical method which is convenient from practical point of view, when a high speed computer is not available.

If we consider the Fjortoft's method from the point of view of harmonic function, we may say as follows: The field of $Z$ is divided into two parts,

$$
Z=Z_{1}+Z_{2}
$$

where $Z_{1}$ and $Z_{2}$ are harmonic functions such as being connected with their wave numbers $\mu_{1}$ and $\mu_{2}$ in the following way

$$
\nabla^{2} Z_{1}=-\mu_{1}^{2} Z_{1}, \quad \nabla^{2} Z_{2}=-\mu_{2}^{2} Z_{2} \text {. }
$$

The instantaneous change of the absolute vorticity in non-divergent and barotropic atmosphere is written

$$
\frac{\partial \eta}{\partial t}=\frac{g}{f} J(\eta, Z)
$$

where

$$
\eta=\frac{g}{f}-\nabla^{2} Z+f
$$

Substituting (1) and (2) into (3), we have

$$
\frac{\partial \eta}{\partial t}=\frac{g}{f}\left(\mu_{1}^{2}-\mu_{2}^{2}\right) J\left(Z_{1}, Z_{2}\right) .
$$

The equation (4) is interpreted in the following two ways. First, we rewrite (4) as follows

$$
\frac{\partial \eta}{\partial t}=\mu_{1}^{2}\left(1-\frac{\mu_{2}^{2}}{\mu_{1}^{2}}\right) V_{2} \cdot \nabla \eta_{1}
$$

where

$$
\boldsymbol{V}_{2}=\frac{g}{f}-K \times \nabla Z_{2} \quad \eta_{1}=\frac{g}{f} \nabla^{2} Z_{1}
$$

The equation (5) means that the instantaneous change of $\eta$ is caused by the advection of $\eta_{1}$ in the field of $Z_{2}$. On the other hand, we have another equation from (4) i.e.,

$$
\frac{\partial \eta}{\partial t}=\mu_{2}^{2}\left(\frac{\mu_{1}^{2}}{\mu_{2}^{2}}-1\right) V_{1} \cdot \nabla \eta_{2}
$$

where

$$
\boldsymbol{V}_{1}=\frac{g}{f} \boldsymbol{K} \times \nabla Z_{1}, \quad \eta_{2}=\frac{g}{f} \nabla^{2} Z_{2}
$$

The equation (6) means that the instantaneous change of $\eta$ is caused by the advection of $\eta_{2}$ in the field of $Z_{1}$. Both (5) and (6) represent the same thing concerning the instantaneous change of $\eta$ while the standpoints of understanding of the role of $Z_{1}$ and $Z_{3}$ are different. The choice of $Z_{1^{\prime}}$ or $Z_{2}$ as the fundamental velocity field depends upon which field has the more stable character with respect to time. Suppose, for instance, the field $Z_{1}$ is more stable than the field $Z_{2}$, it would give us better estimation to use (4) in order to

* Division of Meteorology, Contribution No. 81 
calculate $\eta$ at $t=t_{0}+\Delta t$ from $\eta$ at $t=t_{0}$ by the following simple extrapolation formula,

$$
(\eta)_{t=t_{0}+\Delta t}=(\eta)_{t=t_{0}}+\left(\frac{\partial \eta}{\partial t}\right)_{t=t_{0}} \Delta t
$$

Although Fjortoft did not use harmonic functions, he considered in the same way. As the more stable pattern of $Z$, he used $\bar{Z}$ where bar means the spatial mean value of $Z(x, y)$ which is defined by $\frac{1}{4}\{Z(x+d, y)$ $+Z(x-d, y)+Z(x, y+d)+Z(x, y-d)\}$. In connection with this field he divided $Z$ into two parts

$$
Z=(Z-\bar{Z})+\bar{Z}
$$

The corresponding vorticity $\eta$ is

$$
\eta=\frac{g}{f} \nabla^{2} Z \approx-\frac{4 g}{f d^{2}}(Z-\bar{Z})
$$

Thus the vorticity equation becomes

$$
\frac{\partial \eta}{\partial t}=\frac{g}{f} J(\eta,(Z-\bar{Z})+\bar{Z})=\frac{g}{f} J(\eta, \bar{Z}) .
$$

Another equation which corresponds to this equation, just like relation between (5) and (6), is

$$
\frac{\partial \eta}{\partial t}=\frac{g}{f} J(\eta, Z)
$$

Since $\bar{Z}$ is usuaily more stable than $Z$ with respect to time, (9) gives better results than $(10)$, when we use the extrapolation formula like (7) in the numerical forecasting of $n$. In the present article, we expand $Z$ into double Fourier series with finite components. In this case there are two problems; how many components should be adopted and how to select appropriate harmonic functions among many others. The former problem may be determined from the point of view of the relation between the mesh-size and the scale of disturbances. The latter problem depends upon the character of disturbances, i.e., the stableness of disturbances with respect to time. Concerning the latter problem, we would like to add some comment that the physical consideration mentioned above gives us some clue how to select the appropriate harmonic function. As may be understood from the foregoing discussion, the choice of $Z_{1}$ is better than that of $Z_{2}$ if $Z_{1}$ is more stable than $Z_{2}$ with respect to time. As the fundamental assumption of the problem of this choice, we assume that the large scale patterns do not change so quickly compared with the small scale patterns. Such a consideration enables us to make a choice to select large scale patterns instead of small scale patterns as the selection of the finite number of disturbances. The detailed procedures of the selection of the number and the scale of dis. turbances will be written in the following sections.

\section{§ 2. Numerical Forecasting by Double Fourier Series Method}

The vorticity equation in non-divergent and barotropic atmosphere is written as follows

$$
\frac{\partial}{\partial t} \nabla^{2} Z=J(\eta, Z) \text {. }
$$

Here $Z$ and $\eta$ are the height of isobaric surface of non-divergent level and the absolute vertical vorticity respectively. Using the usual notation, $\eta$ is written in the following way

$$
\eta=h \nabla^{2} Z+f, \quad\left(h=\frac{g}{f} m^{2}\right)
$$

where $m$ and $f$ are the map scale factor and coriolis parameter respectively.

Suppose that the height of the isobaric surface $Z$ may be expanded into harmonic functions, i.e.,

$$
Z=\sum_{n} a_{n} Z_{n},
$$

where $Z_{n}$ is the harmonic function of order $n$. Since $Z_{n z}$ satisfies the following relation

$$
\nabla^{2} Z_{n}=-\mu_{n b}{ }^{2} Z_{n},
$$

where $\mu_{n}$ is the wave number of $Z_{n}$, the substitution of (14) into (11) leads to the following equation

$$
\begin{aligned}
\frac{\partial}{\partial t} \nabla^{2} Z= & \nabla^{2}\left(\frac{\partial Z}{\partial t}\right) \\
= & J\left(f-h \sum_{n} \mu_{n}{ }^{2} a_{n} Z_{n}, \sum_{n} a_{n} Z_{n}\right) \\
= & -h \sum_{i=2}\left(\mu_{1}{ }^{2}-\mu_{i}^{2}\right) a_{1} a_{i} J\left(Z_{1}, Z_{i}\right) \\
& -h \sum_{j=3}\left(\mu_{2}{ }^{3}-\mu_{j}^{2}\right) a_{2} a_{j} J\left(Z_{2}, Z_{j}\right) \\
& -\ldots \ldots \ldots \ldots \ldots \ldots \\
& -\beta \sum a_{n l} \partial Z_{n} .
\end{aligned}
$$

The term of $J\left(Z_{1}, Z_{i}\right)$ in (15) is in proportion to $V_{1} \cdot \nabla \zeta_{i}$, where $V$ and $\zeta_{i}$ are the velocity 
and the vorticity respectively, which are connected with $Z_{1}, Z_{2} \ldots$ as follows:

$$
\boldsymbol{V}_{1}=\frac{g}{f} \boldsymbol{K} \times \nabla_{\nabla} Z_{1}, \quad \zeta_{i}=\frac{g}{f} \nabla^{2} Z_{i} .
$$

Thus the first part on the right hand side in (15) means that the field of $Z_{1}$ makes the vorticitiy of other patterns of $Z_{2}, Z_{3} \ldots$ flow away. In the expression of $J\left(Z_{2}, Z_{j}\right)$ in (15) we see that the field of $Z_{2}$ makes the vorticity of other patterns of $Z_{3}, Z_{4} \ldots$. flow away.

Similar relations may also be expected with the fields of $Z_{3}, Z_{4}, Z_{5}, Z_{6}, \ldots$ In general, the field of $Z_{n}$ makes the vorticities of $Z_{n+1}$, $Z_{n+2}, \ldots$ flow away.

Since $Z_{i}$ is the harmonic function, $J\left(Z_{i}, Z_{j}\right)$ is also the combination of harmonic functions. Such a circumstance makes it easy to integrate (15) with respect to space, Therefore, obtaining $\frac{\partial Z}{\partial t}$ from (15), we make the forecasting of $Z$ as follows

$$
(Z)_{t=t_{0}+\Delta t}=(Z)_{t=t_{0}}+\left(\frac{\partial Z}{\partial t}\right)_{t=t_{0}} \Delta t
$$

As the practical procedure, we expand into the following double Fourier series,

$$
\begin{aligned}
Z & =\sum_{m=0}^{6} \sum_{n=0}^{6} A_{m n} \cos m x \cos n y \\
& +\sum_{m=0}^{6} \sum_{n=1}^{5} B_{m n} \cos m x \sin n y \\
& +\sum_{m=1}^{5} \sum_{n=0}^{6} C_{m n} \sin m x \cos n y \\
& +\sum_{m=1}^{5} \sum_{n=1}^{5} D_{m n} \sin m x \sin n y .
\end{aligned}
$$

The domain where we used for the expansion into the double Fourier series is shown in Fig. 1. From the consideration mentioned above concerning the scale-effect, we put $Z_{1}$, $Z_{2}, Z_{3} \ldots$ as follows,

$$
\begin{aligned}
& a_{0} Z_{0}=A_{00} \quad a_{1} Z_{1}=A_{01} \cos y, \quad a_{2} Z_{2}=B_{01} \sin y \\
& a_{3} Z_{3}=A_{10} \cos x, \quad a_{4} Z_{4}=C_{10} \sin x \\
& a_{5} Z_{5}=A_{11} \cos x \cos y, \ldots \ldots \ldots \ldots \ldots
\end{aligned}
$$

Since $J\left(Z_{i}, Z_{j}\right)$ is a function of sine and cosine, we can write the vorticity equation in the following way

$$
\nabla^{2} \frac{\partial Z}{\partial t}=\nabla^{2} \frac{\partial}{\partial t} \sum a_{n} Z_{n}=\sum_{n} b_{n} Z_{n},
$$

where $b_{n}$ is a function of $\left(A_{01} A_{02} \ldots\right),\left(B_{01}\right.$ $\left.B_{02} \ldots\right),\left(C_{10} C_{11} \ldots\right)$ and $\left(D_{11} D_{12} \ldots\right)$.

Integration of this equation gives the following relation

$$
\frac{\partial a_{n} Z_{n}}{\partial t}=-\frac{b_{n}}{\mu_{n}^{2}} Z_{n}+F_{n},
$$

where $F_{n}$ is the integration function, which satisfies the Laplace equation, i.e.,

$$
\nabla^{2} F_{n}=0 \text {. }
$$

If we write $F=\sum_{n} F_{n}$, the constants which are involved in $F$ are determined from the boundary conditions of $Z$. In order to make the discussion simple, we do not consider the effect from boundaries, so that we neglect $F$. In such a situation, (21) may be written as follows

$$
Z \frac{\partial a_{n}}{\partial t}=-\frac{b_{n}}{\mu_{n}^{2}} Z_{n}
$$

Since $a_{n} Z_{n}$ is one component of $Z$ and similar relations hold for all $n$, we can extrapolate $Z$ with respect to time in the following way

$$
Z\left(t_{0}+\Delta t\right)=\sum a_{n}\left(t_{0}+\Delta t\right) Z_{n},
$$

where

$$
a_{n}\left(t_{0}+\Delta t\right) Z_{n}=a_{n}\left(t_{0}\right) Z_{n}+\left(\frac{\partial a_{n}}{\partial t}\right)_{t \rightarrow t_{0}} Z_{n} \Delta t .
$$

For example, we may write $\frac{\partial a_{n}}{\partial t}$ as follows,

$$
\frac{\partial A_{01}}{\partial t}=R \times\left[\begin{array}{l}
2 A_{10} D_{11}+4 A_{20} D_{21}+3 A_{11} D_{12} \\
+3 A_{12} D_{11}-2 B_{11} C_{10}-4 B_{21} C_{20} \\
-3 B_{11} C_{12}-3 B_{12} C_{11}
\end{array}\right]
$$

where $R=h \frac{\pi^{2}}{L D} ; \quad L$ and $D$ are the longitudinal and latitudinal lengths of the domain respectively, and $A_{i j}, B_{i j}, C_{i j}$ and $D_{i j}$ are the coefficients of the double Fourier series just as may be seen in (18). Here we neglect the terms of wave number larger than 2 . If we take into account up to the wave number 3 , the following equation may be rewritten:

$$
\frac{\partial A_{01}}{\partial t}=R \times\left(\begin{array}{c}
2\left(A_{10} D_{11}-B_{11} C_{10}\right) \\
+3\left(A_{11} D_{12}+A_{13} D_{11}-B_{11} C_{12}\right. \\
\left.-B_{12} C_{11}\right) \\
+4\left(A_{20} D_{21}-B_{21} C_{20}\right) \\
+5\left(A_{12} D_{13}+A_{13} D_{12}-B_{12} C_{13}\right. \\
\left.-B_{13} C_{12}\right) \\
+6\left(A_{30} D_{31}-B_{31} C_{30}\right) \\
+6\left(A_{21} D_{22}+A_{22} D_{21}-B_{21} C_{22}\right. \\
\left.-B_{22} C_{21}\right)
\end{array}\right)
$$


Although it is a complicated calculation to make the schema of the height change for each component of $A_{i j}, B_{i j}, C_{i j}$ and $D_{i j}$, such formula will be useful in order to calculate $\frac{\partial Z}{\partial t}$ at $t=t_{0}$ automatically from the coefficient of $Z$ at $t=t_{0}$. During the process of calculation of $\frac{\partial Z}{\partial t}$ from the value of $Z$, it is not necessary to use the relaxation method.

\section{\$. Discussion of Results}

i) Practical method.

In order to verify the above method, we made 12 hour forecasts ( $\Delta t=12$ hour) at $500-\mathrm{mb}$ level for 1500 GCT 9 Jan., 1953 and for 0300 GCT 11 Jan., 1953. The forecasts for these

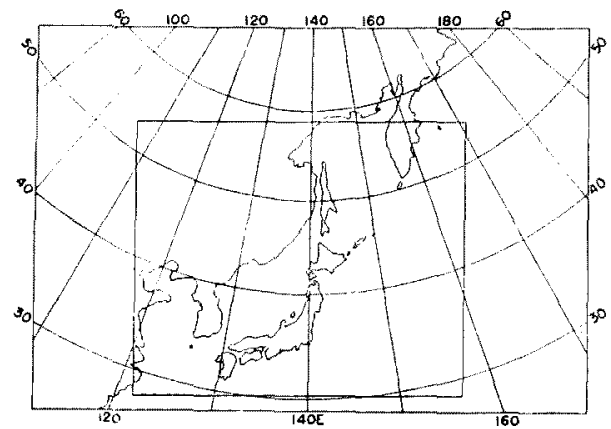

Fig. 1. The map used for calculation. Larger and smaller rectangle in the figure show the area for the calculation and the forecasted area respectively.

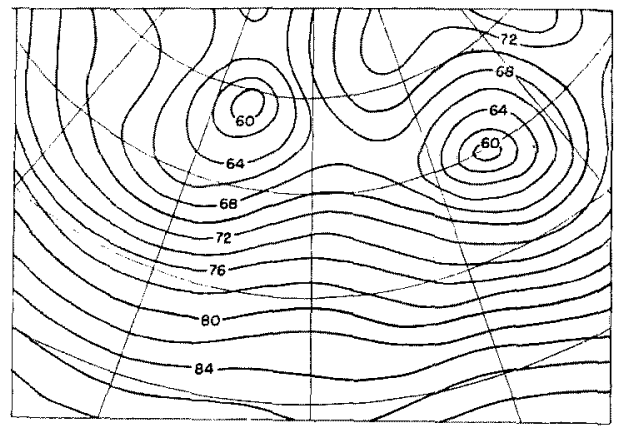

Fig. 2. Initial $500-\mathrm{mb}$ contours for 1500 GCT 9 Jan., 1953.

two days were already made by N.W.P. group in Tokyo [5]. The reason why we select these days is that we can verify our method comparing our results with those which were obtained by N.W.P. group by other methods (by Fjortoft \& Bolin-Charney). In Fig. 1, the rectangle enclosed by solid line shows the area which was selected for the calculation $(6000 \mathrm{~km} \times 4500 \mathrm{~km})$, and the smaller rectangle in the figure shows the forecast area. Fig. 2 shows the weather map for $1500 \mathrm{GCT}$ 9 Jan., which was carefully analysed by $N$. W.P. group in Tokyo. Analysing these data into double Fourier series of 12 components, barotropic changes of contour height at 500 $\mathrm{mb}$ were calculated using double Fourier series method.

\section{ii) Neglection of the waves which have the wave number larger than 2.}

At first we neglected the small scale waves which have the wave number larger than 2 , and calculated the height changes which were caused by the coupling of large scale waves only. Table 1 shows the wave components which were adopted in this case. In other words, we neglected the components which

Table 1. Wave components analysed by double Fourier series from the height pattern on 500 $\mathrm{mb}$, 1500GCT 9 Jan. (Unit in 100 feet). The gothic figures are the selected components used for calculation.

\begin{tabular}{|c|c|c|c|c|c|}
\hline & \multicolumn{5}{|c|}{$A_{m n}$} \\
\hline$m{ }^{n}$ & 0 & 1 & 2 & 3 & 4 \\
\hline 0 & 175.38 & 5.96 & 1.42 & 1.48 & 1.52 \\
\hline 1 & 2.55 & -0.17 & -0.09 & -0.03 & -0.18 \\
\hline 2 & 0.53 & -0.91 & 0.17 & -0.08 & 0.10 \\
\hline & \multicolumn{5}{|c|}{$\mathrm{B}_{m n}$} \\
\hline$m{ }^{n}$ & 1 & 2 & 3 & 4 & \\
\hline 0 & -7.08 & -1.78 & -1.67 & -0.77 & \\
\hline 1 & 1.04 & -0.07 & 0.18 & 0.28 & \\
\hline \multirow[t]{2}{*}{2} & -0.37 & +0.38 & -0.03 & -0.26 & \\
\hline & \multicolumn{5}{|c|}{$C_{m n}$} \\
\hline$m$ & 0 & 1 & 2 & 3 & 4 \\
\hline 1 & 0.65 & -1.66 & 0.30 & 0.19 & 0.21 \\
\hline \multirow[t]{2}{*}{2} & 1.98 & $-\mathbf{1 . 7 1}$ & -0.26 & -0.13 & -0.14 \\
\hline & \multicolumn{5}{|c|}{$D_{m n}$} \\
\hline$m$ & 1 & 2 & 3 & 4 & \\
\hline 1 & -0.67 & -0.67 & -0.07 & -0.44 & \\
\hline 2 & 1.21 & -0.09 & 0.07 & 0.07 & \\
\hline
\end{tabular}


have the wave length shorter than $2250 \mathrm{~km}$ in latitudinal direction and $3000 \mathrm{~km}$ in longitudi. nal direction. Fig. 3 shows the initial contour height of 1500 GCT 9 Jan. being filtered small scale wave components. The results of this forecast ( $\Delta t=12$ hour) for 1500 GCT 9 Jan. may be rather satisfactory. The correlation coefficient is $r=0.66$, which is better than the result calculated by Bolin-Charney's method $(r=0.41)$, and worse than the one by Fjprtoft's method $(r=0.76)$. Fig. 4 shows the calculated and observed change. Adopting $6 \mathrm{hr}$ time interval, nearly same result with Fig. 4 is

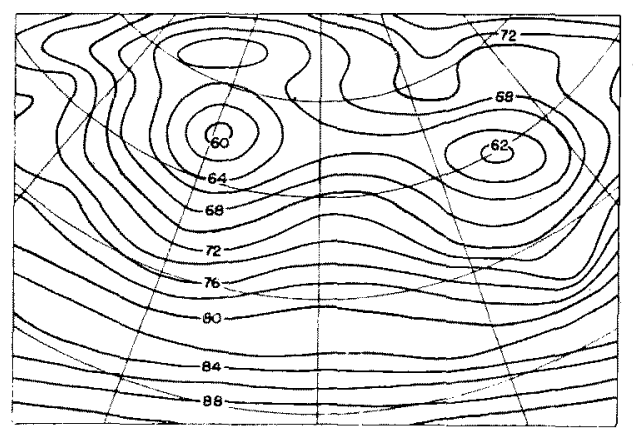

Fig. 3. Initial $500 \mathrm{mb}$ contours for $1500 \mathrm{GCT}$ 9 Jan., 1953, from which small scale waves are filtered.

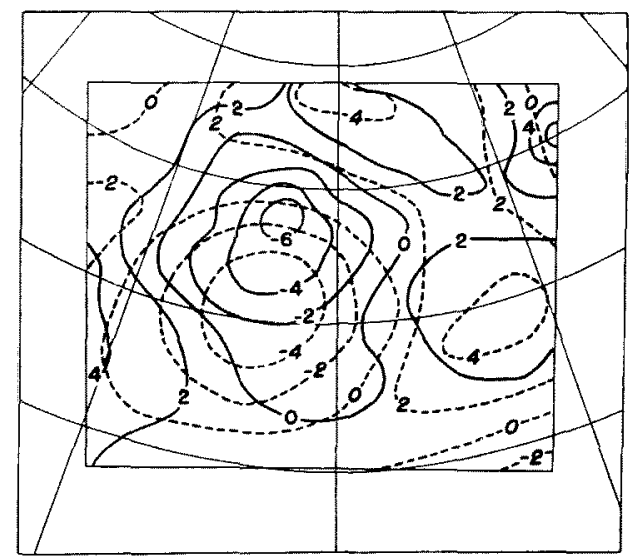

Fig. 4. Forecast of 1500 GCT 11 Jan., 1953. Solid lines show the observed height change for 12 hour of $500-\mathrm{mb}$ contours, and dashed lines the forecasted height change.

1) N.W.P. group in Tokyo forecasted the 12 hour height change for this case using Fjørtoft's method. However, their results were not good. The correlation between observed and calculated height changes was 0.13 when they used 12 hour time interval and 0.53 for 6 hour time interval. obtained. This continuous tendency during 12 hour shows the slow change in larger scale flow patterns, which have the wave number $m, n \leqq 2$.

Next, the 12 hour forecast for $0300 \mathrm{GCT}$ 11 Jan. was made using 6 hour time interval. However, the result was poor. The correlation between the observed and the calculated height change was $0.43^{1}$. In Fig. 5 solid and dashed lines show the observed and calculated height changes respectively. In this case, the initial height pattern and accordingly the pattern of the change of contour height is more complicated than those of $1500 \mathrm{GCT}$ 9 Jan.. Therefore, it may be reasonable to conclude that we must consider the smaller scale wave in order to follow the change of actual height pattern.

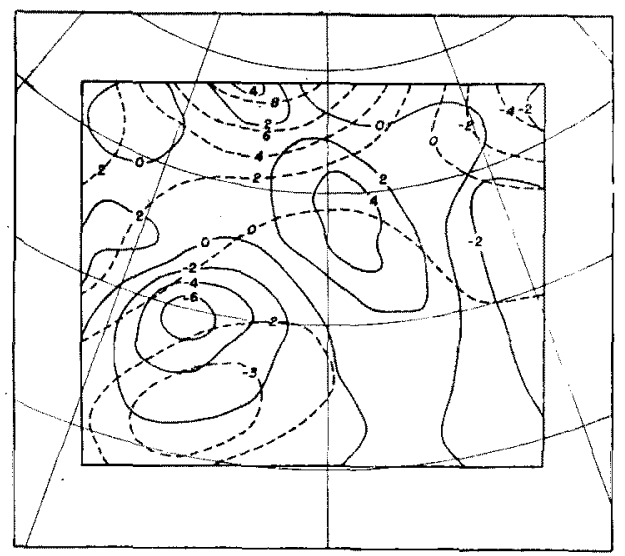

Fig. 5. Forecast of 0300 GCT 11 Jan., 1953. Solid lines show the observed height change for 12 hour of $500-\mathrm{mb}$ contours, and dashed lines the forecasted height change.

\section{iii) Insertion of smaller scale waves.}

Finding that it is necessary to consider the contribution of the smaller scale waves, we took into account of the wave number 3 ; in other words, we neglected the waves which have the wave length shorter than $1500 \mathrm{~km}$ in latitudinal direction and $2000 \mathrm{~km}$ in longitudinal direction. Table 2 shows the wave components which were used for calculation. Owing to the insertion of these waves, the 
Table 2. Wave components used for the revised forecast are shown by the gothic figures (Unit in 100 feet)

\begin{tabular}{|c|c|c|c|c|c|c|c|}
\hline & \multicolumn{4}{|c|}{$A_{m n}$} & \multicolumn{3}{|c|}{$B_{m n}$} \\
\hline$n n$ & 0 & 1 & 2 & 3 & 1 & 2 & 3 \\
\hline 0 & 177.18 & 2.22 & -1.14 & -1.10 & 7.31 & 1. 63 & 1.29 \\
\hline 1 & 3.28 & -1.63 & 0.94 & 0.17 & -0.28 & $8-0.19$ & -0.46 \\
\hline 2 & 0.15 & -0.44 & 0.18 & -0.05 & 1.07 & $7^{\prime}-0.18$ & 0.10 \\
\hline \multirow[t]{2}{*}{3} & -0.21 & 0.39 & -0.25 & 0.02 & 0.03 & $3-0.07$ & -0.09 \\
\hline & \multicolumn{4}{|c|}{$C_{n n n}$} & \multicolumn{3}{|c|}{$D_{n n}$} \\
\hline$m$ & 0 & 1 & 2 & 3 & 1 & 2 & 3 \\
\hline 1 & $-1.04 \mid$ & -2.08 & -0.69 & -0.11 & 0.32 & $2 \quad 1.37$ & 0.76 \\
\hline 2 & 1. 28 & 0.32 & -0.03 & 0.42 & -1.66 & $6-0.12$ & 0.08 \\
\hline 3 & 0.97 & -0.65 & 0.19 & 0.06 & -0.64 & $4 \quad 0$ & 0.02 \\
\hline
\end{tabular}

result was improved remarkably. The correlation between the observed and calculated height change for 12 hour was $0.67^{2}$, which is better than the result ( $r=0.53$ ) obtained by N.W.P. group using the graphical method. Fig. 6 shows the result of this calculation. In this calculation, the advection of small scale vorticity is negiected in order to prevent the accumulation of errors.

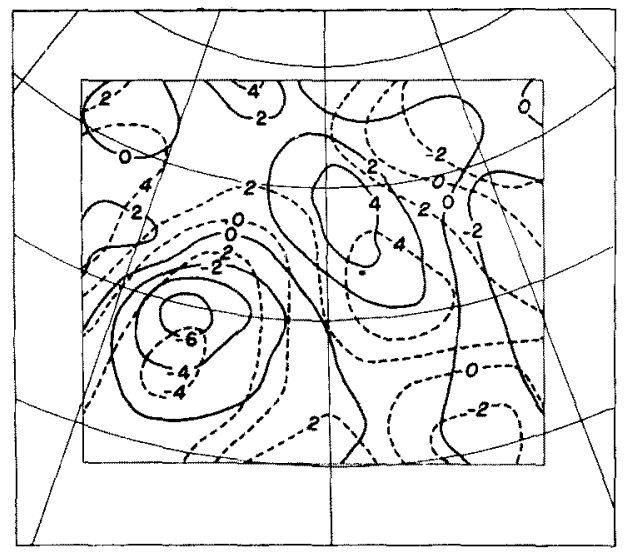

Fig. 6. Revised forecast of 0300 GCT 11 Jan. 1953. Solid lines show the observed height change for 12 hour of $500-\mathrm{mb}$ contours, and dashed lines the forecasted height change.

The equation for the change of contour height of (15) is to be written briefly as follows

$$
\begin{gathered}
\nabla^{2} \frac{\partial Z}{\partial t}=-R \sum_{i} \sum_{j} N_{i j} a_{i} a_{j} J\left(Z_{i}, Z_{j}\right)+\beta \sum_{n} a_{n} \frac{\partial Z_{n}}{\partial x} \\
\left(N_{i j} \equiv \mu_{i}{ }^{2}-\mu_{j}{ }^{2}\right)
\end{gathered}
$$

where $R$ is the coefficient which is defined in $\$ 2$. In the schema for the calculation of height change one example of which is shown in $\S 2$, we can find out many terms which have large $N_{i j}$. These terms may amplify the small errors which are contained in $a_{i}$. Therefore, after testing various filtration, we neglected the terms which have $N_{i j} \mu_{i} \mu_{j}$ larger than 20 . This corresponds to the neglection of the advection of small scale vorticity, i.e. roughly to the neglection of latter two terms in the following equation,

$$
V \cdot \nabla \zeta=\bar{V} \cdot \nabla \bar{\zeta}+V^{\prime} \cdot \nabla \bar{\zeta}+\bar{V} \cdot \nabla \zeta^{\prime}+V \cdot \nabla \zeta^{\prime}
$$

where

$$
\boldsymbol{V}=\overline{\boldsymbol{V}}+\boldsymbol{V}^{\prime \prime}, \quad \zeta=\overline{\boldsymbol{\zeta}}+\boldsymbol{\zeta}^{\prime} .
$$

This filtration was very effective and improved remarkably the result of calculation. Without this neglection, computational instability would occur.

So far, we have realized that our method by double Fourier series was useful and gave us better result than the graphical method.

\section{\$ 4. Discussion about the Influence of Boundaries.}

In the calculation of height change following two assumption was adopted for the sake of simplicity.

i) Periodicity of height pattern.

When we analyse the weather map into double Fourier series, we must assume the periodic repetition of height pattern, which is quite different from the actual situation outside the boundary.

\section{ii) Free boundary condition.}

Same as the graphical solution of relaxation method proposed by Fjortoft, we adopted the free boundary condition for the sake of simplicity.

Owing to these assumption, the effect of erroneous boundary condition penetrates inward. But it is emphasized by many authors that we can avoid this influence by taking the forecast area sufficiently smaller than the region for calculation, because the effect of boundary is propagated by the group velocity which is comparable with the velocity of trough. When the forecast period is shorter

2) Here, we adopted the 6 hour time interval. 
than 24 hours, we can use the present area shown in Fig. 5 for the calculation of height change, while we must, of course, extend this area for the forecast of longer range.

\section{\$ 5. Notes on the Relay-computer.}

Since the relay-computer, FACOM-100, which was made by Fuji Communication Apparatus Manufacturing Company, became to be available, we made the preliminary test of our method by machine. According to our results, a 12-hour forecast by using our method has required the following time:

Expansion of $Z$ into the double Fourier series .................20 minutes Calculation of $\Delta a$, each 1 step .. 30 minutes Composition of the double Fourier series ...................... 20 minutes

As the reference of the characteristics of FACOM-100, the short outline will be written as follows:

\section{Storage:-}

(a) 20 relay-storages-Among them, 4 storages have a printer and another storage has a luminous indicator.

(b) Tape-storages-Each of them has a reader and a perforator.
Both (a) and (b) have the available number part of 8-decimal digits and the power part of 2-decimal digits.

Instructions:-

(a) Three address system

(b) Reading by 2 readers

In the coming year, however, this calculation by relay-computer will be replaced by the electronic computer machine (TAC).

Acknowledgement: The authors wish to express their hearty thanks to Prof. S. Syōno for his kind encouragement throughout this work, to the members of the N.W.P. group in Tokyo for their kind discussions and advices and also to the staff members of Research Section of Fuji C.A.M. Co. for their patience and suggestions in using the relay-computer.

\section{References}

1. Bolin, B. and Charney. J.G. (1951): Tellus 3, 248-257.

2. Charney, J.G., Fj $\phi$ rtoft, R. and von Neumann, J. (1950): Tellus 2, 237-254.

3. Charney, J.G. and Phillips, N.A. (1953): J.M. 10, 71-09.

4. Fjprtoft, R. (1952): Tellus 4, 179-194.

5. N.W.P. Group in Tokyo (1954): Geophys. Mag. 26, 35-51. 